\title{
Advanced Conceptual Models for Unsaturated and Two-Phase Flow in Fractured Rock
}

\author{
Harihar Rajaram \\ Department of Civil, Environmental and \\ Architectural Engineering \\ University of Colorado \\ Campus Box 428 \\ Boulder, CO 80309-0428
}

Advanced Conceptual Models for Unsaturated and Two-Phase Flow in Fractured Rock

Michael J. Nicholl - University of Idaho

Robert J. Glass - Sandia National Laboratories

Harihar Rajaram - University of Colorado

Thomas R. Wood - Idaho National Engineering and Environmental Laboratory

This project was initiated in FY03. As of December 2003, we have accomplished the following: 1) We conducted a more detailed evaluation of the preliminary experiments used to develop our investigative approach. In those experiments, water was invaded at a variety of flow rates into an air-filled, twodimensional analog fracture network. Results demonstrated the critical control that fracture intersections place on two-phase flow in fracture networks. At low flows, capillary and gravitational forces combined to create a narrow pulsing flow structure that spanned the system vertically. At higher flows, viscous forces acted to remove the pulsation; however, the flow structure remained narrow. The intersections acted to impose a narrow "slender ladder" structure on the flowing phase that did not expand with depth, but instead remained focused. A manuscript documenting this effort has been published in Water Resources Research [Glass et al., 2003a]. 2) We initiated a collaborative relationship with a research group at Seoul National University. This group, which is led by Dr. Kang-Kun Lee is also using a combined experimentalnumerical approach to consider DNAPL migration in fracture networks. They are particularly interested in the influence of ambient groundwater flows, making their work complementary to ours. The first fruit of that collaboration is an article demonstrating that modification of an Invasion Percolation algorithm to include gravity and the first-order effects of viscous forces shows good agreement with physical experiments in a simplistic fracture network. Results were published in Geophysical Research Letters [Ji et al., 2003a]. 3) We carried out an extensive review of models for fracture networks. These include models developed from observations of networks on outcrops at several scales and stochastic models that are prevalent in the literature from the 1980s to very recent developments. The results of this review were included as part of a review paper co-authored by Rajaram, which was submitted to Reviews in Geophysics [Molz et al., in press]. 4) We prepared a manuscript based on previous work that will be used to support the development of our new conceptual model(s) for transport in fractured rock. Eight experiments were conducted to evaluate the repeatability of flow under nearly identical conditions and to characterize general patterns in flow behavior. Collected data revealed that flow generally converged to a single fracture in the bottom row of blocks. Periods of pathway switching were observed to be more common than periods with steady, constant flow pathways. We noted the importance of fracture intersections for integrating uniform flow and discharging a "fluid cascade", where water advances rapidly to the next capillary barrier creating a stop and start advance of water through the network. The results of this simple experiment suggest that the interaction of multiple fracture intersections in a network creates flow behavior not generally recognized in popular conceptual and numerical models. A manuscript documenting this effort has been accepted for publication in Vadose Zone Journal [Wood et al., 2003]. 5) Slender transport pathways have been found in laboratory and field experiments within unsaturated fractured rock. We considered the simulation of such structures with a Modified form of Invasion Percolation (MIP). Results show that slender pathways form in fracture networks for a wide range of expected conditions, can be maintained when subsequent matrix imbibition is imposed, and may arise even in the context of primarily matrix flow due to the action of fractures as barriers to inter-matrix block transport. A manuscript documenting this effort has been submitted to Geophysical Research Letters [Glass et al., 2003b]. 